\title{
Dynamics with Low-Level Fractionality
}

\author{
Vasily E. Tarasov ${ }^{1,2}$ and George M. Zaslavsky ${ }^{1,3}$ \\ 1) Courant Institute of Mathematical Sciences, New York University \\ 251 Mercer Street, New York, NY 10012, USA \\ 2) Skobeltsyn Institute of Nuclear Physics, \\ Moscow State University, Moscow 119992, Russia \\ 3) Department of Physics, New York University, \\ 2-4 Washington Place, New York, NY 10003, USA
}

\begin{abstract}
The notion of fractional dynamics is related to equations of motion with one or a few terms with derivatives of a fractional order. This type of equation appears in the description of chaotic dynamics, wave propagation in fractal media, and field theory. For the fractional linear oscillator the physical meaning of the derivative of order $\alpha<2$ is dissipation. In systems with many spacially coupled elements (oscillators) the fractional derivative, along the space coordinate, corresponds to a long range interaction. We discuss a method of constructing a solution using an expansion in $\varepsilon=n-\alpha$ with small $\varepsilon$ and positive integer $n$. The method is applied to the fractional linear and nonlinear oscillators and to fractional Ginzburg-Landau or parabolic equations.
\end{abstract}

PACS: 45.10.Hj; 45.05.+x; 45.50.-j

Keywords: Fractional equations, Fractional oscillator, Ginzburg-Landau equation

\section{Introduction}

It became clear in the last decade that many physical processes can be adequately described by equations that consist of derivatives of fractional order. In a fairly short period of time, 
the list of such applications is long and the areas of applications are broad. Even in a concise form, the applications include material sciences [1, 2], chaotic dynamics [3], quantum theory $[4,5,6,7]$, physical kinetics $[3,8,9,10]$, fluids and plasma physics $[11,12,13,14]$, and many others physical topics related to anomalous diffusion, wave propagation [15], and transport theory (see more in reviews $[3,16])$. Since the fractional calculus has a fairly long history, the approaches for solutions of corresponding equations are also enormously rich. Let us mention some of these approaches that are related more specifically to this paper: probabilistic basis and interpretation of the fractional kinetics $[17,18,9,19]$, dissipative interpretation of the fractional derivative [20, 21, 22, 23], Green's function method [24, 25], etc.

This paper is motivated by a lack of methods that permit an explicit analysis of the equations with fractional derivatives that describe some important physical processes. Particularly, the difficulties are due to the absence of characteristic scales (sometimes such processes are called multiscaling) and the difficulty of direct estimates. In typical physical situations the "level of fractality" is low, i.e. the order of fractional derivatives $\alpha$ of the corresponding terms of the dynamical equations slightly deviates from an integer value $n$ (in the considered cases $n=1$ or 2). This recalls a possibility to use an expansion over the small parameter $\varepsilon=n-\alpha$ that we call an $\varepsilon$-expansion. We develop a construction that is applied to linear and nonlinear fractional oscillators. Particularly, for the linear fractional oscillator (LFO) the obtained expansion can be compared to the exact one. As a more complicated example, we consider a solution of the fractional Ginzburg-Landau equation (FGL) introduced in [26, 27] (see also [28]).

The basic description of the $\varepsilon$-expansion is given in Sec. 2 for different fractional derivatives. In Sec. 3, we consider examples of the $\varepsilon$-expansion application to linear and nonlinear oscillators. In Sec. 4 , we consider large $t$ asymptotics $(t \rightarrow \infty)$. Application of the $\varepsilon$-expansion to the Landau-Ginzburg or to the parabolic nonlinear equation is given in Sec. 5. Some technical details are the Appendices 1, 2. 


\section{Description of the $\varepsilon$-expansion}

\subsection{Caputo fractional derivative of order $\alpha=2-\varepsilon$}

The fractional derivative has different definitions [29, 30], and exploiting any of them depends on the kind of the problems, initial (boundary) conditions, and the specifics of the considered physical processes. The classical definition is the so-called Riemann-Liouville derivative $[29,30$, $31]$

$$
\begin{aligned}
& { }_{a} \mathcal{D}_{t}^{\alpha} f(x)=\frac{1}{\Gamma(n-\alpha)} \frac{\partial^{n}}{\partial x^{n}} \int_{a}^{x} \frac{f(z) d z}{(x-z)^{\alpha-n+1}}, \\
& { }_{t} \mathcal{D}_{b}^{\alpha} f(x)=\frac{(-1)^{n}}{\Gamma(n-\alpha)} \frac{\partial^{n}}{\partial x^{n}} \int_{x}^{b} \frac{f(z) d z}{(z-x)^{\alpha-n+1}},
\end{aligned}
$$

where $n-1<\alpha<n$. Due to reasons, concerning the initial conditions, it is more convenient to use the Caputo fractional derivatives $[2,32,33]$. Its main advantage is that the initial conditions take the same form as for integer-order differential equations.

The left Caputo fractional derivative $[2,32,33]$ is defined by the equation

$$
D^{\alpha} f(t)={ }_{0}^{C} D_{t}^{\alpha} f(t)=\frac{1}{\Gamma(n-\alpha)} \int_{0}^{t} \frac{f^{(n)}(\tau)}{(t-\tau)^{\alpha+1-n}} d \tau,
$$

where $n-1<\alpha<n$, and $f^{(n)}(\tau)=d^{n} f(\tau) / d \tau^{n}$. For $n=2$,

$$
D^{2-\varepsilon} f(t)=\frac{1}{\Gamma(\varepsilon)} \int_{0}^{t} \frac{f^{(2)}(\tau)}{(t-\tau)^{1-\varepsilon}} d \tau, \quad 0<\varepsilon<1 .
$$

This presentation is not convenient in the limit $\varepsilon \rightarrow 0$, since

$$
\frac{1}{\Gamma(\varepsilon)}=\varepsilon+O\left(\varepsilon^{2}\right)
$$

and we present (2) and (3) in the form [25]:

$$
D^{\alpha} f(t)=\frac{f^{(n)}(0) t^{n-\alpha}}{\Gamma(n-\alpha+1)}+\frac{1}{\Gamma(n-\alpha+1)} \int_{0}^{t} f^{(n+1)}(\tau)(t-\tau)^{n-\alpha} d \tau, \quad n-1<\alpha \leq n,
$$

or for $n=2$,

$$
D^{2-\varepsilon} f(t)=\frac{f^{(2)}(0) t^{\varepsilon}}{\Gamma(1+\varepsilon)}+\frac{1}{\Gamma(1+\varepsilon)} \int_{0}^{t} f^{(3)}(\tau)(t-\tau)^{\varepsilon} d \tau .
$$

Let us consider first the case

$$
\varepsilon t \ll 1 \text {. }
$$


We can use the expansion

$$
\begin{gathered}
\frac{1}{\Gamma(1+\varepsilon)}(t-\tau)^{\varepsilon}=\frac{1}{\Gamma(1+\varepsilon)} e^{\varepsilon \ln (t-\tau)}= \\
=1+\varepsilon(\gamma+\ln (t-\tau))+\varepsilon^{2}\left(\frac{1}{2} \ln ^{2}(t-\tau)+\gamma \ln (t-\tau)+\frac{\gamma^{2}}{2}-\frac{\pi^{2}}{12}\right)+\ldots,
\end{gathered}
$$

where $\tau<t$, and $\gamma=0.5772156649 \ldots$ is a constant. As the result, we obtain

$$
D^{2-\varepsilon} f(t)=f^{(2)}(t)+\varepsilon\left(f^{(2)}(0) \ln (t)+\gamma f^{(2)}(t)+\int_{0}^{t} f^{(3)}(\tau) \ln (t-\tau) d \tau\right)+\ldots
$$

that expresses the fractional derivative in a form of the perturbation to the second derivative, when $\varepsilon \ll 1$ :

$$
D^{2-\varepsilon} f(t)=f^{(2)}(t)+\varepsilon D_{1}^{2} f(t)+\ldots
$$

where $D_{1}^{2}$ is defined as:

$$
D_{1}^{2} f(t)=f^{(2)}(0) \ln (t)+\gamma f^{(2)}(t)+\int_{0}^{t} f^{(3)}(\tau) \ln (t-\tau) d \tau
$$

Note that, the limit $\varepsilon \rightarrow 0$ in equation (9) gives the correct expansion

$$
\lim _{\varepsilon \rightarrow 0} D^{2-\varepsilon} f(t)=f^{(2)}(t)
$$

Another useful comments is that the $3 \mathrm{~d}$ derivative $f^{(3)}(t)$ should exist in order to use the correction of order $\varepsilon$ in (9).

As a simple example of application of formula (9), consider the $\alpha$-derivative of $t^{2}$ and $t^{3}$ :

$$
\begin{gathered}
D^{2-\varepsilon} t^{2}=2+2 \varepsilon \gamma+\ldots \\
D^{2-\varepsilon} t^{3}=6 t+\varepsilon[6(\gamma-1) t+6 t \ln (t)]+\ldots
\end{gathered}
$$

From the well-known relation

$$
D^{\alpha} t^{\beta}=\frac{\Gamma(\beta+1)}{\Gamma(\beta-\alpha+1)} t^{\beta-\alpha}
$$

we get the exact results

$$
\begin{aligned}
D^{2-\varepsilon} t^{2} & =\frac{2}{\Gamma(1+\varepsilon)} t^{\varepsilon}, \\
D^{2-\varepsilon} t^{3} & =\frac{6}{\Gamma(2+\varepsilon)} t^{1+\varepsilon} .
\end{aligned}
$$

Expansions of (14) for $\varepsilon t \ll 1$ coincide with (12). 


\subsection{Caputo fractional derivative of order $\alpha=1-\varepsilon$.}

To consider the limit $\alpha \rightarrow 1$, let us put $n=1$ in (5):

$$
D^{1-\varepsilon} f(t)=\frac{f^{(1)}(0) t^{\varepsilon}}{\Gamma(1+\varepsilon)}+\frac{1}{\Gamma(1+\varepsilon)} \int_{0}^{t} f^{(2)}(\tau)(t-\tau)^{\varepsilon} d \tau
$$

Similarly to $n=2$, consider the case $\varepsilon t \ll 1$, and use the expansion (8). It gives

$$
D^{1-\varepsilon} f(t)=f^{(1)}(t)+\varepsilon D_{1}^{1} f(t)+\ldots
$$

where $D_{1}^{1} f(t)$ is

$$
D_{1}^{1} f(t)=f^{(1)}(0) \ln (t)+\gamma f^{(1)}(t)+\int_{0}^{t} f^{(2)}(\tau) \ln (t-\tau) d \tau
$$

Note that, if we consider the limit $\varepsilon \rightarrow 0$, we get

$$
\lim _{\varepsilon \rightarrow 0} D^{1-\varepsilon} f(t)=f^{(1)}(t)
$$

\subsection{Riesz fractional derivatives of order $\alpha=2-\varepsilon$}

The Riesz fractional derivative of order $\alpha$ is defined by

$$
\frac{d^{\alpha}}{d|x|^{\alpha}} f(x)=-\frac{1}{2 \cos (\pi \alpha / 2)}\left(\mathcal{D}_{+}^{\alpha} f(x)+\mathcal{D}_{-}^{\alpha} f(x)\right),
$$

where $\alpha \neq 1,3,5 \ldots$, and $\mathcal{D}_{ \pm}^{\alpha}$ are Riemann-Liouville fractional derivatives

$$
\begin{aligned}
& \mathcal{D}_{+}^{\alpha} f(x)=\frac{1}{\Gamma(n-\alpha)} \frac{\partial^{n}}{\partial x^{n}} \int_{-\infty}^{x} \frac{f(z) d z}{(x-z)^{\alpha-n+1}}, \\
& \mathcal{D}_{-}^{\alpha} f(x)=\frac{(-1)^{n}}{\Gamma(n-\alpha)} \frac{\partial^{n}}{\partial x^{n}} \int_{x}^{\infty} \frac{f(z) d z}{(z-x)^{\alpha-n+1}} .
\end{aligned}
$$

Substitution of Eqs. (20) into Eq. (19) gives

$$
\frac{d^{\alpha}}{d|x|^{\alpha}} f(x)=-\frac{1}{2 \cos (\pi \alpha / 2) \Gamma(n-\alpha)} \frac{\partial^{n}}{\partial x^{n}}\left(\int_{-\infty}^{x} \frac{f(z) d z}{(x-z)^{\alpha-n+1}}+\int_{x}^{\infty} \frac{f(z) d z}{(z-x)^{\alpha-n+1}}\right) .
$$

For the order $\alpha=2-\varepsilon$,

$$
\frac{d^{\alpha}}{d|x|^{\alpha}} f(x)=\frac{1}{2 \cos (\pi \varepsilon / 2) \Gamma(\varepsilon)} \frac{\partial^{2}}{\partial x^{2}}\left(\int_{-\infty}^{x} \frac{f(z) d z}{(x-z)^{1-\varepsilon}}+(-1)^{n} \int_{x}^{\infty} \frac{f(z) d z}{(z-x)^{1-\varepsilon}}\right) .
$$

Using the condition $f( \pm \infty)=0$, we get

$$
\int_{-\infty}^{x} \frac{f(z) d z}{(x-z)^{1-\varepsilon}}=\int_{-\infty}^{x} f(z)(x-z)^{\varepsilon-1} d z=\frac{1}{\varepsilon} \int_{-\infty}^{x} f^{(1)}(z)(x-z)^{\varepsilon} d z,
$$




$$
\int_{x}^{\infty} \frac{f(z) d z}{(z-x)^{1-\varepsilon}}=\int_{x}^{\infty} f(z)(z-x)^{\varepsilon-1} d z=-\frac{1}{\varepsilon} \int_{x}^{\infty} f^{(1)}(z)(z-x)^{\varepsilon} d z .
$$

These relations and equation $\varepsilon \Gamma(\varepsilon)=\Gamma(1+\varepsilon)$ allows us to present (21) in the form

$$
\frac{d^{\alpha}}{d|x|^{\alpha}} f(x)=\frac{1}{2 \cos (\pi \varepsilon / 2) \Gamma(1+\varepsilon)} \frac{\partial^{2}}{\partial x^{2}}\left(\int_{-\infty}^{x} f^{(1)}(z)(x-z)^{\varepsilon} d z-\int_{x}^{\infty} f^{(1)}(z)(z-x)^{\varepsilon} d z\right) .
$$

For the case $\varepsilon x \ll 1$, we use

$$
\frac{(x-z)^{\varepsilon}}{\cos (\pi \varepsilon / 2) \Gamma(1+\varepsilon)}=1+\varepsilon[\gamma+\ln (x-z)]+\ldots
$$

to obtain

$$
\frac{d^{2-\varepsilon}}{d|x|^{2-\varepsilon}} f(x)=f^{(2)}(x)+\varepsilon D_{1 R}^{2} f(x)+\ldots
$$

where

$$
D_{1 R}^{2} f(x)=\gamma f^{(2)}(x)+\frac{1}{2} \int_{0}^{\infty}\left[f^{(3)}(x-y)-f^{(3)}(x+y)\right] \ln (y) d y .
$$

A general comments to this section is that all results (11), (17) and (27) are valid under restriction $\varepsilon t \ll 1$. The expansion of Riemann-Liouville derivative is considered in Appendix 1 . For the asymptotic $t \rightarrow \infty$, and

$$
\varepsilon t \gg 1
$$

another expansion is necessary. The corresponding results will be considered in section 4 .

\section{Linear and nonlinear fractional oscillator}

\section{$3.1 \quad \varepsilon$-expansion for linear oscillator}

A linear fractional oscillator (LFO) is defined by the equation

$$
D^{\alpha} x(t)+\omega^{2} x(t)=0,
$$

where $\omega$ is dimensionless "frequency", $\alpha=2-\varepsilon, 0<\varepsilon \ll 1$, and $D^{2-\varepsilon}$ is Caputo fractional derivative that allows us to use the usual initial conditions [25]. LFO is an object of numerous investigations $[20,21,22,23,34,35,36,37,38]$ because of different applications.

The exact solution of Eq. (29) for $1<\alpha<2$ is [20, 21]:

$$
x(t)=x(0) E_{\alpha, 1}\left(-\omega^{2} t^{\alpha}\right)+t x^{\prime}(0) E_{\alpha, 2}\left(-\omega^{2} t^{\alpha}\right),
$$


where

$$
E_{\alpha, \beta}(z)=\sum_{k=0}^{\infty} \frac{z^{k}}{\Gamma(\alpha k+\beta)}
$$

is the generalized two-parameter Mittag-Leffler function, and

$$
E_{\alpha}(z)=E_{\alpha, 1}(z)=\sum_{k=0}^{\infty} \frac{z^{k}}{\Gamma(\alpha k+1)}
$$

is one-parameter, or simply, Mittag-Leffler function [39, 40].

Existence of an exact solution of (29) in the form (30) permits a comparison with the $\varepsilon$ expansion

$$
x(t)=x_{0}(t)+\varepsilon x_{1}(t)+\ldots
$$

constructed below. The equation for $x_{0}(t)$ and the corresponding initial conditions are

$$
x_{0}^{\prime \prime}(t)+\omega^{2} x_{0}(t)=0, \quad x_{0}(0)=x(0), \quad x_{0}^{\prime}(0)=x^{\prime}(0),
$$

with the solution

$$
x_{0}(t)=x(0) \cos (\omega t)+\left(x^{\prime}(0) / \omega\right) \sin (\omega t) .
$$

The equation for $x_{1}(t)$ and the initial conditions are

$$
x_{1}^{\prime \prime}(t)+\omega^{2} x_{1}(t)+D_{1}^{2} x_{0}(t)=0, \quad x_{1}(0)=0, \quad x_{1}^{\prime}(0)=0,
$$

where $D_{1}^{2} x_{0}(t)$ is defined by $(11)$ :

$$
D_{1}^{2} x_{0}(t)=x_{0}^{\prime \prime}(0) \ln (t)+\gamma x_{0}^{\prime \prime}(t)+\int_{0}^{t} x_{0}^{(3)}(\tau) \ln (t-\tau) d \tau .
$$

Substitution of Eq. (35) into Eq. (37) gives

$$
\begin{aligned}
& D_{1} x_{0}(t)=-\omega^{2} x(0) \ln (t)-\gamma \omega^{2}\left[x(0) \cos (\omega t)+\left(x^{\prime}(0) / \omega\right) \sin (\omega t)\right]+ \\
& +\omega^{3} x(0) \int_{0}^{t} \sin (\omega \tau) \ln (t-\tau) d \tau-\omega^{2} x^{\prime}(0) \int_{0}^{t} \cos (\omega \tau) \ln (t-\tau) d \tau .
\end{aligned}
$$

The first and second integrals can be calculated, and we have the relations

$$
\begin{gathered}
\int_{0}^{t} \sin (\omega \tau) \ln (t-\tau) d \tau= \\
=\omega^{-1}\left(\ln (t)+\cos (\omega t)[\ln (\omega)+\gamma-\operatorname{Ci}(\omega \mathrm{t})]+\sin (\omega t)\left[\frac{\gamma+1}{2}-\frac{\pi}{4}-\operatorname{Si}(\omega \mathrm{t})\right]\right),
\end{gathered}
$$


and

$$
\begin{gathered}
\int_{0}^{t} \cos (\omega \tau) \ln (t-\tau) d \tau= \\
=\omega^{-1}\left(\cos (\omega t)\left[\frac{\gamma+1}{2}-\frac{\pi}{4}-\operatorname{Si}(\omega \mathrm{t})\right]+\sin (\omega t)[-\ln (\omega)-\gamma+\operatorname{Ci}(\omega \mathrm{t})]\right) .
\end{gathered}
$$

Here $\mathrm{Ci}(\mathrm{t})$ and $\mathrm{Si}(\mathrm{t})$ are sine and cosine integral functions [42] respectively:

$$
\operatorname{Si}(\mathrm{t})=\int_{0}^{\mathrm{t}} \frac{\sin (\mathrm{x})}{\mathrm{x}} \mathrm{dx}=\operatorname{si}(\mathrm{t})+\pi / 2, \quad \operatorname{Ci}(\mathrm{t})=-\int_{\mathrm{t}}^{\infty} \frac{\cos (\mathrm{x})}{\mathrm{x}} \mathrm{dx}=-\operatorname{ci}(\mathrm{t}) .
$$

As the result, Eq. (36) transforms into

$$
\begin{gathered}
x_{1}^{\prime \prime}(t)+\omega^{2} x_{1}(t)+\left[\omega^{2} x(0) \cos (\omega t)+\omega v_{0} \sin (\omega t)\right](\ln (\omega)-\operatorname{Ci}(\omega t))+ \\
+\left[\omega^{2} x(0) \sin (\omega t)-\omega v_{0} \cos (\omega t)\right]\left(\frac{\gamma+1}{2}-\frac{\pi}{4}-\operatorname{Si}(\omega t)\right)=0 .
\end{gathered}
$$

To simplify the calculations consider $x(0)=1, x^{\prime}(0)=0$, and $\omega=1$. Then $x_{0}(t)=\cos (t)$, and

$$
x_{1}^{\prime \prime}(t)+x_{1}(t)-\cos (t) \operatorname{Ci}(\mathrm{t})-\sin (\mathrm{t}) \operatorname{Si}(\mathrm{t})+\left(\frac{\gamma+1}{2}-\frac{\pi}{4}\right) \sin (\mathrm{t})=0 .
$$

The solution of this equation is

$$
\begin{aligned}
x_{1}(t) & =\cos (t) \int_{0}^{t}\left[\left(\frac{\gamma+1}{2}-\frac{\pi}{4}\right)\left[1-\cos ^{2}(u)\right]-\sin (u) \cos (u) \operatorname{Ci}(\mathrm{u})-\operatorname{Si}(\mathrm{u})+\operatorname{Si}(\mathrm{u}) \cos ^{2}(\mathrm{u})\right] d u+ \\
& +\sin (t) \int_{0}^{t}\left[-\left(\frac{\gamma+1}{2}-\frac{\pi}{4}\right) \sin (u) \cos (u)+\cos ^{2}(u) \operatorname{Ci}(\mathrm{u})+\sin (\mathrm{u}) \cos (\mathrm{u}) \operatorname{Si}(\mathrm{u})\right] d u,
\end{aligned}
$$

which after integration takes the form

$$
x_{1}(t)=-\frac{t}{2}[\sin (t) \operatorname{Ci}(\mathrm{t})+\cos (\mathrm{t}) \operatorname{Si}(\mathrm{t})]-\frac{1}{2}+\frac{1}{2} \cos (t) .
$$

Finally, Eq. (33) provides the solution

$$
x(t)=\cos (t)-\frac{\varepsilon}{2}(1-\cos (t)+t[\sin (t) \operatorname{Ci}(\mathrm{t})+\cos (\mathrm{t}) \operatorname{Si}(\mathrm{t})])+\ldots
$$

The term, that is proportional to $\varepsilon$, can be considered as a correction to the solution $x(t)=\cos (t)$ due to the fractional derivative of order $\alpha=2-\varepsilon$.

Let us compare this result with the exact solution for FLO. The decomposition of (30) is [20]:

$$
x(t)=x(0)\left[f_{\alpha, 0}(t)+g_{\alpha, 0}(t)\right]+t x^{\prime}(0)\left[f_{\alpha, 1}(t)+g_{\alpha, 1}(t)\right],
$$


where

$$
\begin{gathered}
f_{\alpha, k}(t)=\frac{(-1)^{k}}{\pi} \int_{0}^{\infty} e^{-r t} \frac{r^{\alpha-1-k} \sin (\pi \alpha)}{r^{2 \alpha}+2 r^{\alpha} \cos (\pi \alpha)+1} d r \\
g_{\alpha, k}(t)=\frac{2}{\alpha} e^{t \cos (\pi / \alpha)} \cos [t \sin (\pi / \alpha)-\pi k / \alpha], \quad(k=0,1) .
\end{gathered}
$$

For the initial conditions $x(0)=1$, and $x^{\prime}(0)=0$ :

$$
x(t)=E_{\alpha}\left(-t^{\alpha}\right)=f_{\alpha, 0}(t)+g_{\alpha, 0}(t) .
$$

Using $\alpha=2-\varepsilon$ and the condition $\varepsilon t \ll 1$, we get

$$
\begin{gathered}
f_{\alpha, 0}(t)=-\frac{\varepsilon}{2}\left(1+t \sin (t) \operatorname{Ci}(\mathrm{t})+\mathrm{t} \cos (\mathrm{t}) \operatorname{Si}(\mathrm{t})-\mathrm{t} \frac{\pi}{2} \cos (\mathrm{t})\right)+\ldots, \\
g_{\alpha, 0}(t)=\cos (t)+\varepsilon\left(\frac{1}{2}-\frac{\pi}{4} t\right) \cos (t)+\ldots
\end{gathered}
$$

Substitution of (49) into (48) gives Eq. (45).

\subsection{Fractional oscillator in phase space}

The variety of definitions of fractional derivatives causes some inconvenience in their applications to different physical problems. Particularly, this comment concerns the phase space definition for the fractional dynamics, which is not uniquely defined in the general case. As an example, consider the fractional Caputo derivative of order $\alpha=2-\varepsilon$ that can be defined as

$$
D^{\alpha}=D^{2-\varepsilon}=J^{\varepsilon} D^{2},
$$

where $J^{\varepsilon}$ is a fractional integration of order $\varepsilon$. Therefore, we can use the relation

$$
D^{2-\varepsilon}=J^{\varepsilon} D^{2}=J^{\varepsilon} D^{1} D^{1}=\left(J^{\varepsilon} D^{1}\right) D^{1}=D^{1-\varepsilon} D^{1}
$$

to represent the fractional equation

$$
D^{2-\varepsilon} x(t)=F(x(t))
$$

as a system of two equations

$$
D^{1} x(t)=p(t), \quad D^{1-\varepsilon} p(t)=F(x(t)),
$$

where first equation has derivative of integer order. 
In general, we have an inequality

$$
D^{1-\varepsilon_{1}} D^{1-\varepsilon_{2}} \neq D^{2-\left(\varepsilon_{1}+\varepsilon_{2}\right)},
$$

where $D^{1-\varepsilon}=J^{\varepsilon} D^{1}$, i.e., the composition doesn't exist for the Caputo derivatives

$$
D^{1-\varepsilon_{1}} D^{1-\varepsilon_{2}} x(t)=D^{2-\left(\varepsilon_{1}+\varepsilon_{2}\right)} x(t)+\frac{1}{\Gamma\left(\varepsilon_{1}+\varepsilon_{2}\right)} t^{\varepsilon_{1}+\varepsilon_{2}-1} x^{\prime}(0),
$$

and the operations $D^{1}$ and $J^{\varepsilon}$ do not commute:

$$
D^{1} J^{\varepsilon} x(t)=J^{\varepsilon} D^{1} x(t)+\frac{t^{\varepsilon-1}}{\Gamma(\varepsilon)} x(0) .
$$

The symmetrized fractional equation

$$
D^{1-\varepsilon / 2}\left[D^{1-\varepsilon / 2} x(t)\right]=F(x(t)),
$$

is not the same as (52), since

$$
D^{1-\varepsilon / 2}\left[D^{1-\varepsilon / 2} x(t)\right]=D^{2-\varepsilon} x(t)+\frac{t^{\varepsilon-1}}{\Gamma(\varepsilon)} x^{\prime}(0) .
$$

Eq. (57) is equivalent to (52) for $x^{\prime}(0)=0$. Consider Eq. (57), and represent it as a system of fractional equations

$$
D^{1-\varepsilon / 2} x(t)=p(t), \quad D^{1-\varepsilon / 2} p(t)=F(x(t))
$$

where both equations are fractional equations. Using Eq. (16), we can rewrite (59) as

$$
x^{\prime}(t)+(\varepsilon / 2) D_{1}^{1} x(t)+\ldots=p(t), \quad p^{\prime}(t)+(\varepsilon / 2) D_{1}^{1} p(t)+\ldots=F(x(t))
$$

and consider their solution in the form

$$
x(t)=x_{0}(t)+\varepsilon x_{1}(t)+\ldots ., \quad p(t)=p_{0}(t)+\varepsilon p_{1}(t)+\ldots
$$

As the result, we get a system of equations:

$$
\begin{gathered}
x_{0}^{\prime}(t)=p_{0}(t), \quad p_{0}^{\prime}(t)=F\left(x_{0}(t)\right), \\
x_{1}^{\prime}(t)+D_{1}^{1} x_{0}(t)=p_{1}(t), \quad p_{1}^{\prime}(t)+D_{1}^{1} p_{0}(t)=\left(\frac{\partial F(x)}{\partial x}\right)_{x=x_{0}} x_{1}(t) .
\end{gathered}
$$

For the initial conditions $x_{0}(0)=1, p_{0}(0)=0$, and $F(x)=-x$, the perturbation terms $D_{1}^{1} x_{0}$ and $D_{1}^{1} p_{0}$ in $(62)$ are defined as

$$
D_{1}^{1} x_{0}(t)=-\gamma \sin (t)-\int_{0}^{t} \cos (\omega \tau) \ln (t-\tau) d \tau
$$




$$
D_{1}^{1} p_{0}(t)=-\ln (t)-\gamma \cos (t)+\int_{0}^{t} \sin (\omega \tau) \ln (t-\tau) d \tau,
$$

and leads Eq. (62) to the form

$$
\begin{aligned}
& x_{1}^{\prime}(t)+\cos (t) \operatorname{Si}(\mathrm{t})-\sin (\mathrm{t}) \operatorname{Ci}(\mathrm{t})-\left[\frac{\gamma+1}{2}-\frac{\pi}{4}\right] \cos (\mathrm{t})=\mathrm{p}_{1}(\mathrm{t}), \\
& p_{1}^{\prime}(t)-\cos (t) \mathrm{Ci}(\mathrm{t})-\sin (\mathrm{t}) \operatorname{Si}(\mathrm{t})+\left[\frac{\gamma+1}{2}-\frac{\pi}{4}\right] \sin (\mathrm{t})=-\mathrm{x}_{1}(\mathrm{t})
\end{aligned}
$$

with solution

$$
\begin{gathered}
x_{1}(t)=-t \sin (t) \operatorname{Ci}(\mathrm{t})-\mathrm{t} \cos (\mathrm{t}) \operatorname{Si}(\mathrm{t})+\cos (\mathrm{t})-1, \\
p_{1}(t)=-\sin (t) \mathrm{Ci}(\mathrm{t})-\cos (\mathrm{t}) \operatorname{Si}(\mathrm{t})-\mathrm{t} \cos (\mathrm{t}) \mathrm{Ci}(\mathrm{t})+\mathrm{t} \sin (\mathrm{t}) \operatorname{Si}(\mathrm{t})-\sin (2 \mathrm{t})-\sin (\mathrm{t}) .
\end{gathered}
$$

This solution describes the phase space evolution of FLO for $\varepsilon t \ll 1$.

\subsection{Nonlinear fractional oscillator}

Let us consider a fractional nonlinear oscillator that is defined by the equation

$$
D^{2-\varepsilon} x(t)-x(t)+2 x^{3}(t)=0,
$$

where $0<\varepsilon \ll 1$, and $D^{2-\varepsilon}$ is a Caputo fractional derivative. For the soliton-type solution

$$
x_{0}(t)=\frac{1}{\cosh (t)}=\operatorname{sech}(t),
$$

the correction of the order $\varepsilon$ can be found similar to (45):

$$
x_{1}^{\prime \prime}(t)+\left[6 \operatorname{sech}^{2}(t)-1\right] x_{1}(t)+D_{1}^{2} \operatorname{sech}(t)=0,
$$

where

$$
D_{1}^{2} \operatorname{sech}(t)=-\ln (t)+\gamma \frac{\cosh ^{2}(t)-2}{\cosh ^{3}(t)}+\int_{0}^{t} \frac{\sinh ^{2}(\tau)\left[\cosh ^{2}(\tau)-6\right]}{\cosh ^{4}(\tau)} \ln (t-\tau) d \tau .
$$

The solution $x_{1}(t)$ of equation (69) can be derived numerically. As the result we can get the corrections to the solution $x_{0}(t)$. 


\section{Asymptotic behavior for large $t$}

\subsection{Asymptotic representation of fractional derivative}

In the previous sections, we consider the intermediate time asymptotic limited by the condition $\varepsilon t \ll 1$. In this section, we consider the opposite condition $t \rightarrow \infty$.

It is known $[32,25]$, that the Laplace transform of the Caputo fractional derivative is

$$
\int_{0}^{\infty} e^{-s t}\left[D^{\alpha} x(t)\right] d t=s^{\alpha} X(s)-\sum_{k=0}^{n-1} s^{\alpha-k-1} x^{(k)}(0)
$$

where $n-1<\alpha \leq n$, and $X(s)$ is a Laplace transform of $x(t)$ :

$$
X(s)=\int_{0}^{\infty} e^{-s t} x(t) d t
$$

Note that formula (70) involves the initial conditions $x^{(k)}(0)$ with integer derivatives $x^{(k)}$. Therefore we can put the initial conditions in the usual way. The functions $x(t)$ satisfy the condition

$$
\int_{0}^{\infty} e^{-s t}|x(t)|<\infty
$$

For $1<\alpha \leq 2$, Eq. (70) has the form

$$
\int_{0}^{\infty} e^{-s t}\left[D^{\alpha} x(t)\right] d t=s^{\alpha} X(s)-s^{\alpha-1} x(0)-s^{\alpha-2} x^{\prime}(0) .
$$

Inversion of (73) gives

$$
D^{\alpha} x(t)=\frac{1}{2 \pi i} \int_{B r} e^{s t}\left[s^{\alpha} X(s)-s^{\alpha-1} x(0)-s^{\alpha-2} x^{\prime}(0)\right] d s .
$$

where $B r$ denotes the Bromwich contour, i.e., a line from $c-i \infty$ to $c+i \infty$, where $\operatorname{Re}(s)=c$, and the contour is taken to the right of all singularities in order to insure condition (72). Closing the contour to the right will yield $x(t)=0$ for $t<0$. For non-integer $\alpha$ the power function $s^{\alpha}$ is uniquely defined as $s^{\alpha}=|s|^{\alpha} \exp [\mathrm{i} \arg (\mathrm{s})]$, with $-\pi<\arg (s)<\pi$, that is in the complex $s$-plane cut along the negative real semi-axis.

The asymptotic expansion $(\varepsilon t \gg 1)$ can be formally obtained by the expanding $X(s)$ in powers of $s$, and then inverting term-by-term. The asymptotic for large $t$ corresponds to small s. Consider the Taylor series

$$
X(s)=\sum_{k=0}^{\infty} \frac{1}{k !} X^{(k)}(0) s^{k}, \quad|s|<1 .
$$


Substitution of (75) into (74) yields

$$
\begin{gathered}
D^{\alpha} x(t)=-x(0) \frac{1}{2 \pi i} \int_{B r} e^{s t} s^{\alpha-1} d s-x^{\prime}(0) \frac{1}{2 \pi i} \int_{B r} e^{s t} s^{\alpha-2} d s+ \\
+\sum_{k=0}^{\infty} \frac{1}{k !} X^{(k)}(0) \frac{1}{2 \pi i} \int_{B r} e^{s t} s^{\alpha+k} d s .
\end{gathered}
$$

Using the analytical continuation of

$$
\frac{1}{2 \pi i} \int_{B r} s^{\beta} d s=\frac{t^{-\beta-1}}{\Gamma(-\beta)}, \quad \operatorname{Re}(\beta)<0
$$

to the half-plane $\operatorname{Re}(\beta) \geq 0$, we arrive to the equation

$$
D^{\alpha} x(t)=-\frac{x(0) t^{-\alpha}}{\Gamma(1-\alpha)}-\frac{x^{\prime}(0) t^{1-\alpha}}{\Gamma(2-\alpha)}+\sum_{k=0}^{\infty} \frac{X^{(k)}(0) t^{-\alpha-k-1}}{k ! \Gamma(-\alpha-k)}, \quad(t \rightarrow \infty), \quad 1<\alpha<2
$$

with the leading asymptotic term

$$
D^{2-\varepsilon} x(t) \approx-x(0) \frac{t^{-2+\varepsilon}}{\Gamma(-1+\varepsilon)}-x^{\prime}(0) \frac{t^{-1+\varepsilon}}{\Gamma(\varepsilon)} \approx \varepsilon x(0) t^{-2+\varepsilon}-\varepsilon x^{\prime}(0) t^{-1+\varepsilon}, \quad(\varepsilon t \gg 1) .
$$

It is seen from (79) that for arbitrary small $x^{\prime}(0)$ the first term can be neglected for sufficiently large $t$.

\subsection{Asymptotics for linear fractional oscillator}

Let us consider some applications of the results of the previous section. For the linear fractional oscillator

$$
D^{\alpha} x(t)+\omega^{2} x(t)=0,
$$

with $\alpha=2-\varepsilon$, we obtain from (79),

$$
x(t) \approx \frac{x(0)}{\omega^{2}} \frac{t^{-2+\varepsilon}}{\Gamma(-1+\varepsilon)}+\frac{x^{\prime}(0)}{\omega^{2}} \frac{t^{-1+\varepsilon}}{\Gamma(\varepsilon)} \approx-\varepsilon \frac{x(0)}{\omega^{2}} t^{-2+\varepsilon}+\varepsilon \frac{x^{\prime}(0)}{\omega^{2}} t^{-1+\varepsilon}, \quad(\varepsilon t \gg 1) .
$$

This result can be also derived from the asymptotic of the Mittag-Leffler function using the exact solution of $(80)$,

$$
x(t)=x(0) E_{\alpha}\left(-\omega^{2} t^{\alpha}\right)+x^{\prime}(0) t E_{\alpha, 2}\left(-\omega^{2} t^{\alpha}\right) .
$$

Let us use the following integral representation

$$
E_{\alpha}(z)=\frac{1}{2 \pi i} \int_{H a} \frac{\xi^{\alpha-1} e^{\xi}}{\xi^{\alpha}-z} d \xi
$$


where $\mathrm{Ha}$ denotes the Hankel path, a loop which starts from $-\infty$ along the lower side of the negative real axis, encircles the circular disc $|\xi| \leq|z|^{1 / \alpha}$ in the positive direction, and ends at $-\infty$ along the upper side of the negative real axis. By the replacement $\xi^{\alpha} \rightarrow \xi$ Eq. (83) transforms into $[25,44]$ :

$$
E_{\alpha}(z)=\frac{1}{2 \pi i \alpha} \int_{\gamma(a, \delta)} \frac{e^{\xi^{1 / \alpha}}}{\xi-z} d \xi, \quad(1<\alpha<2),
$$

where $\pi \alpha / 2<\delta<\min \{\pi, \pi \alpha\}$. The contour $\gamma(a, \delta)$ consists of two rays $S_{-\delta}=\{\arg (\xi)=$ $-\delta,|\xi| \geq a\}$ and $S_{+\delta}=\{\arg (\xi)=+\delta,|\xi| \geq a\}$, and a circular $\operatorname{arc} C_{\delta}=\{|\xi|=1,-\delta \leq \operatorname{arc}(\xi) \leq$ $\delta$. Let us denote the region on the left from $\gamma(a, \delta)$ as $G^{-}(a, \delta)$. Then [44]:

$$
E_{\alpha}(z)=-\sum_{n=1}^{\infty} \frac{z^{-n}}{\Gamma(1-\alpha n)}, \quad z \in G^{-}(a, \delta), \quad(|z| \rightarrow \infty),
$$

and $\delta \leq|\arg (z)| \leq \pi$. In our case, $z=-\omega^{2} t^{\alpha}, \arg (z)=\pi$, and

$$
E_{\alpha}\left(-\omega^{2} t^{\alpha}\right) \approx \frac{t^{-\alpha}}{\omega^{2} \Gamma(1-\alpha)} \approx-\varepsilon \frac{1}{\omega^{2}} t^{-2+\varepsilon} .
$$

In a similar way, we get

$$
E_{\alpha, 2}\left(-\omega^{2} t^{\alpha}\right) \approx \frac{t^{-\alpha}}{\omega^{2} \Gamma(2-\alpha)} \approx \varepsilon \frac{1}{\omega^{2}} t^{-2+\varepsilon}
$$

We arrive at the asymptotic result (81) that exhibits an algebraic decay for $t \rightarrow \infty$. This algebraic decay is the most important effect of the non-integer derivative in the considered fractional equations, contrary to the exponential decay of the usual damped-oscillation and linear relaxation phenomena.

\subsection{Nonlinear fractional oscillator}

The results of the previous section can be extended to the nonlinear fractional oscillator. Consider the equation

$$
D^{2-\varepsilon} x(t)-x(t)+x^{3}(t)=0,
$$

and try to find its solution in the form

$$
x(t)=x_{0}(t)+\varepsilon x_{1}(t)+\ldots
$$

For $\varepsilon=0, x_{0}(t)$ satisfies the equation

$$
x_{0}^{\prime \prime}(t)-x_{0}(t)+x_{0}^{3}(t)=0 .
$$


Consider a particular solution

$$
x_{0}(t)=\sqrt{2} \operatorname{sech}(t),
$$

and its Laplace transform [41]:

$$
X(s)=\int_{0}^{\infty} x_{0}(t) e^{-s t} d t=\frac{1}{\sqrt{2}} \Psi\left(\frac{s+3}{4}\right)-\frac{1}{\sqrt{2}} \Psi\left(\frac{s+1}{4}\right),
$$

where $\Psi(z)$ is the digamma function

$$
\Psi(z)=\frac{\Gamma^{\prime}(z)}{\Gamma(z)}=\frac{d}{d z} \ln [\Gamma(z)]
$$

For $z \ll 1$

$$
\Psi(z)=-\frac{1}{z}-\gamma+\frac{\pi^{2}}{6} z+O\left(z^{2}\right)
$$

and therefore

$$
X(s)=\frac{\pi}{2}+\frac{1}{8}\left[\Psi\left(1, \frac{3}{4}\right)-\Psi\left(1, \frac{1}{4}\right)\right] s+O\left(s^{2}\right), \quad(s \rightarrow 0),
$$

where

$$
\Psi(n, z)=\frac{d^{n} \Psi(z)}{d z^{n}},
$$

is the $n$-th polygamma function, and

$$
\frac{1}{8}\left[\Psi\left(1, \frac{3}{4}\right)-\Psi\left(1, \frac{1}{4}\right)\right]=-1.831931188 \ldots
$$

Substitution of (79) into Eq. (88) gives

$$
x^{3}(t)-x(t)-\frac{\sqrt{2}}{\Gamma(1-\alpha)} t^{-\alpha} \approx 0 .
$$

The leading asymptotic term is

$$
x(t) \approx \frac{A(t)}{6 \Gamma(1-\alpha)}+\frac{2 \Gamma(1-\alpha)}{A(t)}
$$

where

$$
A(t)=\left(108 \sqrt{2} t^{-\alpha}+\left[-12 \Gamma^{2}(1-\alpha)+162 t^{-2 \alpha}\right]^{1 / 2}\right)^{1 / 3} \approx c_{1}+c_{2} t^{-\alpha} \quad(t \rightarrow \infty)
$$

and

$$
c_{1}=2^{1 / 3}(-3 \Gamma(1-\alpha))^{1 / 6}, \quad c_{2}=2^{5 / 6} 18(-3 \Gamma(1-\alpha))^{-1 / 3} .
$$




\section{Fractional Ginzburg-Landau (FGL) equation}

\subsection{Appearance of fractional derivatives in Ginzburg-Landau equation}

Let us recall the appearance of the nonlinear parabolic equation [45, 46, 47, 48], and the FGL suggested in Ref. [26] (see also [27, 28]). Consider wave propagation in some media and present the wave vector $\mathbf{k}$ in the form

$$
\mathbf{k}=\mathbf{k}_{0}+\kappa=\mathbf{k}_{0}+\kappa_{\|}+\kappa_{\perp}
$$

where $\mathbf{k}_{0}$ is the unperturbed wave vector and subscripts $(\|, \perp)$ are taken respectively to the direction of $\mathbf{k}_{0}$. A symmetric dispersion law $\omega=\omega(k)$ for $\kappa \ll k_{0}$ can be written as

$$
\omega(k)=\omega(|\mathbf{k}|) \approx \omega\left(k_{0}\right)+v_{g}\left(|\mathbf{k}|-k_{0}\right)+\frac{1}{2} v_{g}^{\prime}\left(|\mathbf{k}|-k_{0}\right)^{2},
$$

where

$$
v_{g}=\left(\frac{\partial \omega}{\partial k}\right)_{k=k_{0}}, \quad v_{g}^{\prime}=\left(\frac{\partial^{2} \omega}{\partial k^{2}}\right)_{k=k_{0}}
$$

and

$$
|\mathbf{k}|=\left|\mathbf{k}_{0}+\boldsymbol{\kappa}\right|=\sqrt{\left(\mathbf{k}_{0}+\kappa_{\|}\right)^{2}+\kappa_{\perp}^{2}} \approx k_{0}+\kappa_{\|}+\frac{1}{2 k_{0}} \kappa_{\perp}^{2} .
$$

Substitution of (105) into (103) gives

$$
\omega(k) \approx \omega_{0}+v_{g} \kappa_{\|}+\frac{v_{g}}{2 k_{0}} \kappa_{\perp}^{2}+\frac{v_{g}^{\prime}}{2} \kappa_{\|}^{2}
$$

where $\omega_{0}=\omega\left(k_{0}\right)$. Expression (106) in the dual space ("momentum representation") corresponds to the following equation in the coordinate space

$$
i \frac{\partial Z}{\partial t}=\omega_{0} Z-i v_{g} \frac{\partial Z}{\partial x}-\frac{v_{g}}{2 k_{0}} \Delta_{\perp} Z-\frac{v_{g}^{\prime}}{2} \Delta_{\|} Z
$$

with respect to the field $Z=Z(t, x, y, z)$, where $x$ is along $\mathbf{k}_{0}$, and we use the operator correspondence between the dual space and usual space-time:

$$
\begin{gathered}
\omega(k) \longleftrightarrow i \frac{\partial}{\partial t}, \quad \kappa_{\|} \longleftrightarrow-i \frac{\partial}{\partial x} \\
\left(\boldsymbol{\kappa}_{\perp}\right)^{2} \longleftrightarrow-\Delta_{\perp}=-\frac{\partial^{2}}{\partial y^{2}}-\frac{\partial^{2}}{\partial z^{2}}, \quad\left(\boldsymbol{\kappa}_{\|}\right)^{2} \longleftrightarrow-\Delta_{\|}=-\frac{\partial^{2}}{\partial x^{2}}
\end{gathered}
$$


A generalization to the nonlinear case can be carried out similarly to (106) through a nonlinear dispersion law dependence on the wave amplitude:

$$
\omega=\omega\left(k,|Z|^{2}\right) \approx \omega(k, 0)+b|Z|^{2}=\omega(|\mathbf{k}|)+b|Z|^{2}
$$

with some constant $b=\partial \omega\left(k,|Z|^{2}\right) / \partial|Z|^{2}$ at $|Z|=0$. In analogy to (107), we obtain from (106), and (108):

$$
i \frac{\partial Z}{\partial t}=\omega\left(k_{0}\right) Z-i v_{g} \frac{\partial Z}{\partial x}-\frac{v_{g}}{2 k_{0}} \Delta_{\perp} Z-\frac{v_{g}^{\prime}}{2} \Delta_{\|} Z+b|Z|^{2} Z .
$$

This equation is known as the nonlinear parabolic equation [45, 46, 47, 48]. The change of variables from $(t, x, y, z)$ to $\left(t, x-v_{g} t, y, z\right)$ gives

$$
-i \frac{\partial Z}{\partial t}=\frac{v_{g}}{2 k_{0}} \Delta_{\perp} Z+\frac{v_{g}^{\prime}}{2} \Delta_{\|} Z-\omega\left(k_{0}\right) Z-b|Z|^{2} Z
$$

that is also known as the nonlinear Schrödinger (NLS) equation.

Wave propagation in a media with fractal properties can be easily generalized by rewriting the dispersion law (106), (109) in the following way [26]:

$$
\omega\left(k,|Z|^{2}\right)=\omega\left(k_{0}, 0\right)+v_{g} \kappa_{\|}+g_{1}\left(\boldsymbol{\kappa}_{\perp}^{2}\right)^{\alpha / 2}+g_{2}\left(\boldsymbol{\kappa}_{\|}^{2}\right)^{\beta / 2}+b|Z|^{2}, \quad(1<\alpha, \beta<2)
$$

with new constants $g_{1}, g_{2}$.

Using the connection between Riesz fractional derivative and its Fourier transform [29]

$$
\left(-\Delta_{\perp}\right)^{\alpha / 2} \longleftrightarrow\left(\boldsymbol{\kappa}_{\perp}^{2}\right)^{\alpha / 2}, \quad\left(-\Delta_{\|}\right)^{\beta / 2} \longleftrightarrow\left(\boldsymbol{\kappa}_{\|}^{2}\right)^{\beta / 2}
$$

we obtain from (112)

$$
i \frac{\partial Z}{\partial t}=-i v_{g} \frac{\partial Z}{\partial x}+g_{1}\left(-\Delta_{\perp}\right)^{\alpha / 2} Z+g_{2}\left(-\Delta_{\|}\right)^{\beta / 2} Z+\omega_{0} Z+b|Z|^{2} Z,
$$

where $Z=Z(t, x, y, z)$. By changing the variables from $(t, x, y, z)$ to $(t, \xi, y, z), \xi=x-v_{g} t$, and using

$$
\left(-\Delta_{\|}\right)^{\beta / 2}=\frac{\partial^{\beta}}{\partial|x|^{\beta}}=\frac{\partial^{\beta}}{\partial|\xi|^{\beta}},
$$

we obtain from (114) equation

$$
i \frac{\partial Z}{\partial t}=g_{1}\left(-\Delta_{\perp}\right)^{\alpha / 2} Z+g_{2}\left(-\Delta_{\|}\right)^{\beta / 2} Z+\omega_{0} Z+b|Z|^{2} Z
$$

that can be called the fractional nonlinear parabolic equation. For $g_{2}=0$ we get the nonstationary FGL equation (fractional NLS equation) suggested in [26]. Let us comment on the 
physical structure of (116). The first term on the right-hand side is related to wave propagation in a media with fractal properties. The fractional derivative can also appear as a result of ray chaos $[15,49]$ or due to a superdiffusive wave propagation (see also the discussion in $[3,15]$ and corresponding references therein). Other terms on the right-hand-side of Eqs. (114), and (116) correspond to wave interaction due to the nonlinear properties of the media. Thus, Eq. (116) can describe fractal processes of self-focusing and related issues.

We may consider one-dimensional simplifications of (116), i.e.,

$$
i \frac{\partial Z}{\partial t}=g_{2} \frac{\partial^{\beta} Z}{\partial|\xi|^{\beta}}+\omega_{0} Z+b|Z|^{2} Z
$$

where $Z=Z(t, \xi), \xi=x-v_{g} t$, or the equation

$$
i \frac{\partial Z}{\partial t}=g_{1} \frac{\partial^{\alpha} Z}{\partial|z|^{\alpha}}+\omega_{0} Z+b|Z|^{2} Z
$$

where $Z=Z(t, z)$. We can reduce (118) to the case of a propagating wave

$$
Z=Z\left(z-v_{g} t\right) \equiv Z(\eta)
$$

Then (118) becomes

$$
g_{1} \frac{d^{\alpha} Z}{d|\eta|^{\alpha}}+c \frac{d Z}{d \eta}+\omega_{0} Z+b Z^{3}=0, \quad \eta=z-v_{g} t
$$

for real $Z(\eta)$, and $c=i v_{g}$. This equation takes the form of a fractional generalization of the Ginzburg-Landau equation (FGL), when $v_{g}=0$. Eq. (120) differs from the fractional Burgers equation $[50,51]$ in the structure of the nonlinear term. Nevertheless, an analysis similar to $[50,51]$ may be performed to obtain some estimates on the solution [26].

\section{$5.2 \quad \varepsilon$-expansion for the FGL equation}

Let us consider $\varepsilon$-expansion for a particular case of equation (118) when the time dependence can be excluded by the replacement

$$
Z(t, z)=e^{i a t} \bar{Z}(z)
$$

It gives the FGL equation

$$
g \frac{d^{\alpha} \bar{Z}}{d|z|^{\alpha}}+\bar{\omega} \bar{Z}+b \bar{Z}^{3}=0, \quad g=g_{1}, \quad \bar{\omega}=\omega_{0}+a c
$$


for real field $\bar{Z}=\bar{Z}(z)$. Again, let us search for a solution in the form

$$
\bar{Z}(z)=\bar{Z}_{0}(z)+\varepsilon \bar{Z}_{1}(z)+\ldots
$$

and

$$
\frac{d^{2-\varepsilon} \bar{Z}}{d|z|^{2-\varepsilon}}=\frac{d^{2} \bar{Z}}{d z^{2}}+\varepsilon D_{1}^{2} \bar{Z}+\ldots
$$

similar to section 3.1. In zero approximation $\bar{Z}(z)=\bar{Z}_{0}(z)$ which satisfies the equation

$$
g \frac{d^{2} \bar{Z}_{0}}{d z^{2}}+\bar{\omega} \bar{Z}_{0}+b \bar{Z}_{0}^{3}=0
$$

As in section 3.3 consider a particular solution of (125):

$$
\bar{Z}_{0}(z)=|2 \bar{\omega} / b|^{1 / 2} \operatorname{sech}\left(|\bar{\omega} / g|^{1 / 2} z\right)
$$

In the first approximation

$$
g \frac{d^{2} \bar{Z}_{1}}{d z^{2}}+\left(3 b \bar{Z}_{0}^{2}+\bar{\omega}\right) \bar{Z}_{1}+g D_{1}^{2} \bar{Z}_{0}=0
$$

where $\bar{Z}_{0}=\bar{Z}_{0}(z)$ is defined in (126), and

$$
D_{1}^{2} \bar{Z}_{0}(z)=\gamma \bar{Z}_{0}^{(2)}(z)+\frac{1}{2} \int_{0}^{\infty} d y \ln (y)\left[\bar{Z}_{0}^{(3)}(z-y)-\bar{Z}_{0}^{(3)}(z+y)\right]
$$

The derivatives in (128) are

$$
\begin{gathered}
\bar{Z}_{0}^{(2)}(z)=\left|\frac{2 \bar{\omega}}{b}\right|^{1 / 2} \frac{A^{2}\left(\cosh ^{2}(A z)-2\right)}{\cosh ^{3}(A z)}, \\
\bar{Z}_{0}^{(3)}(z)=\left|\frac{2 \bar{\omega}}{b}\right|^{1 / 2} \frac{A^{3} \sinh (A z)\left(6-\cosh ^{2}(A z)\right)}{\cosh ^{4}(A z)},
\end{gathered}
$$

where $A=|\bar{\omega} / g|^{1 / 2}$. Eq. (127) with respect to $\bar{Z}_{1}(z)$ has only the integer derivatives and can be solved approximately or numerically. The asymptotics $z \rightarrow \infty$ for (122) is discussed in Appendix 2.

\section{Conclusion}

Equations with fractional derivatives can be considered as a convenient tool to model different physical processes with dissipation, persistent memory, and long range interaction. To work with such type equations, one needs different approximate schemes such as perturbation theory, 
asymptotics expansions, etc. In our paper we develop $\varepsilon$-expansion for the cases when the order of fractional derivatives $\alpha$ can be presented as $\alpha=n-\varepsilon, n=1,2$, and $\varepsilon \ll 1$. This expansion is not uniform with respect to $t \gg 1$, and two cases $\varepsilon t \ll 1$, $\varepsilon t \gg 1$, are very different. We demonstrate how these expansions work for some examples that have exact a solution (fractional linear oscillator) and examples when the $\varepsilon$-expansion is not trivial (fractional nonlinear oscillator, fractional Ginzburg-Landau equation). Fractional derivatives are not defined uniquely, and for different types of derivatives the $\varepsilon$-expansion is different.

\section{Acknowledgments}

Authors thank M.F. Shlesinger for valuable remarks. This work was supported by the Office of Naval Research, Grant No. N00014-02-1-0056, the U.S. Department of Energy Grant No. DEFG02-92ER54184, and the NSF Grant No. DMS-0417800. VET thanks the Courant Institute of Mathematical Sciences for support and kind hospitality.

\section{Appendix 1: $\varepsilon$-expansion for Riemann-Liouville frac- tional derivatives of order $\alpha=2-\varepsilon$}

From the equation

$$
{ }_{x}^{C} D_{b}^{\alpha} f(x)={ }_{x} \mathcal{D}_{b}^{\alpha}\left(f(x)-\sum_{k=0}^{n-1} \frac{x^{k}}{k !} f^{(k)}(b)\right),
$$

where $n-1<\alpha<n$, follows

$$
{ }_{0} \mathcal{D}_{t}^{\alpha} f(t)={ }_{0} D_{t}^{\alpha} f(t)+\sum_{k=0}^{n-1} \frac{t^{k-\alpha}}{\Gamma(k-\alpha+1)} f^{(k)}(0),
$$

or for $n=2, \alpha=2-\varepsilon$

$$
{ }_{0} \mathcal{D}_{t}^{2-\varepsilon} f(t)={ }_{0} D_{t}^{2-\varepsilon} f(t)+\frac{t^{-2+\varepsilon}}{\Gamma(-1+\varepsilon)} f(0)+\frac{t^{-1+\varepsilon}}{\Gamma(\varepsilon)} f^{(1)}(0) .
$$

For $\varepsilon t \ll 1$, we use the expansions

$$
\begin{aligned}
\frac{t^{-2+\varepsilon}}{\Gamma(-1+\varepsilon)} & =t^{-2}\left(-\varepsilon+\varepsilon^{2}[1-\gamma-\ln (t)]+\ldots\right), \\
\frac{t^{-1+\varepsilon}}{\Gamma(\varepsilon)} & =t^{-1}\left(\varepsilon+\varepsilon^{2}[\gamma+\ln (t)]+\ldots\right) .
\end{aligned}
$$


From Eq. (132) and (9), we obtain

$$
{ }_{0} \mathcal{D}_{t}^{2-\varepsilon} f(t)=f^{(2)}(t)+\varepsilon \mathcal{D}_{1}^{2} f+\ldots
$$

where

$$
\mathcal{D}_{1}^{2} f(t)=-t^{-2} f(0)+t^{-1} f^{(1)}(0)+f^{(2)}(0) \ln (t)+\gamma f^{(2)}(t)+\int_{0}^{t} f^{(3)}(\tau) \ln (t-\tau) d \tau .
$$

Expressions (135), (136) provides the $\varepsilon$-expansion for Riemann-Liouville fractional derivative.

\section{Appendix 2: Large $x$ asymptotics for FGL equation}

In section 4 , we consider the asymptotic $t \rightarrow \infty$ for Caputo derivative. Let us consider a modified equation (118), where we replace the Riesz derivative $\partial^{\alpha} Z / \partial|z|^{\alpha}$ by the Caputo derivative $D^{\alpha} Z$ :

$$
i \frac{\partial Z}{\partial t}=g D^{\alpha} Z+\omega_{0} Z+b|Z|^{2} Z
$$

where $Z=Z(t, z), g=g_{1}$, and the boundary condition is $Z_{z}^{(1)}(t, 0)=0$. Again, exclude the term with first derivative, as in (121), by the replacement

$$
Z(t, z)=e^{i a t} \bar{Z}(z)
$$

It gives modified FGL equation

$$
g D^{\alpha} \bar{Z}+\bar{\omega} \bar{Z}+b|\bar{Z}|^{2} \bar{Z}=0
$$

where $\bar{\omega}=\omega_{0}+a c$, and $\bar{Z}^{(1)}(0)=0$. Substitution of (79) into (139) yields

$$
b|\bar{Z}|^{2} \bar{Z}+\bar{\omega} \bar{Z}-g \frac{z^{-\alpha}}{\Gamma(1-\alpha)} \bar{Z}(0) \approx 0 .
$$

Using representation

$$
\bar{Z}(z)=R(z) e^{i \phi(z)}
$$

where $R(z)=|\bar{Z}(z)|$ is real and $\phi(z)=\arg (\bar{Z})$, Eq. (140) can be rewritten as

$$
b R^{3}(z)+\bar{\omega} R(z)-g \frac{z^{-\alpha}}{\Gamma(1-\alpha)} R(0) e^{i[\phi(0)-\phi(z)]} \approx 0 .
$$

If the constants $b, \bar{\omega}, g$ are real, then

$$
b R^{3}(z)+\bar{\omega} R(z) \pm g \frac{z^{-\alpha}}{\Gamma(1-\alpha)} R(0) \approx 0, \quad \phi(z)=\phi(0)+\pi n
$$


where $n$ is an integer. The solution of (143) is

$$
R_{1}(z) \approx \pm \varepsilon \frac{g R(0)}{\bar{\omega} \Gamma(1-\alpha)} z^{-\alpha} \approx \mp \varepsilon(g / \bar{\omega}) R(0) z^{-2+\varepsilon}, \quad(z \rightarrow \infty)
$$

for $\bar{\omega} \neq 0$, or

$$
R_{2}(z) \approx \pm \frac{g^{1 / 3} z^{-\alpha / 3}}{[b \Gamma(1-\alpha)]^{1 / 3}} R^{1 / 3}(0) \approx \mp \varepsilon^{1 / 3}(g / b)^{1 / 3} z^{(-2+\varepsilon) / 3} R^{1 / 3}(0)
$$

for $\bar{\omega}=0, b \neq 0$. Finally,

$$
Z_{1}(t, z) \approx \pm \frac{g z^{-\alpha} e^{i a t}}{\bar{\omega} \Gamma(1-\alpha)} Z(0,0) \approx \mp \varepsilon(g / \bar{\omega}) z^{-2+\varepsilon} e^{i a t} Z(0,0),
$$

and

$$
Z_{2}(t, z) \approx \pm \frac{g^{1 / 3} z^{-\alpha / 3} e^{i a t}}{[b \Gamma(1-\alpha)]^{1 / 3}} R^{-2 / 3}(0) Z(0,0) \approx \mp \varepsilon^{1 / 3}(g / b)^{1 / 3} z^{(-2+\varepsilon) / 3} R^{-2 / 3}(0) e^{i a t} Z(0,0) .
$$

\section{References}

[1] R. Hilfer (Ed.), Applications of Fractional Calculus in Physics (World Scientific, Singapore, 2000)

[2] M. Caputo, Elasticita e Dissipazione, (Zanichelli, Bologna, 1969)

[3] G.M. Zaslavsky, Phys. Rep. 371 (2002) 461-580.

[4] N. Laskin, Phys. Rev. E 66 (2002) 056108; Chaos 10 (2000) 780-790; Phys. Rev. E 62 (2000) 3135-3145; Phys. Lett. A 268 (2000) 298-305.

[5] M. Naber, J. Math. Phys. 45 (2004) 3339-3352.

[6] J.P. Krisch, J. Math. Phys. 46 (2005) 042506.

[7] E. Goldfain, Chaos, Solitons and Fractals 22 (2004) 513-520; 19 (2004) 1023-1030.

[8] G.M. Zaslavsky, M.A. Edelman, Physica D 193 (2004) 128-147.

[9] A.I. Saichev, G.M. Zaslavsky, Chaos 7 (1997) 753-764.

[10] G.M. Zaslavsky, Physica D 76 (1994) 110-122.

[11] B.A. Carreras, V.E. Lynch, G.M. Zaslavsky, Physics of Plasmas 8 (2001) 5096-5103.

[12] R.R. Nigmatullin, Phys. Status Solidi B 133 (1986) 425-430; Theor. Math. Phys. 90 (1992) $242-251$.

[13] A. Le Mehaute, R.R. Nigmatullin, L. Nivanen, Fleches du temps et geometric fractale (Hermes, Paris, 1998) 
[14] V.E. Tarasov, Physics of Plasmas 12 (2005) 082106; Ann. Phys. 318 (2005) 286-307; Phys. Lett. A 336 (2005) 167-174 (cond-mat/0506137); Chaos 15 (2005) 023102.

[15] V.V. Zosimov, L.M. Lyamshev, Uspekni Fizicheskih Nauk 165 (1995) 361-402.

[16] R. Metzler, J. Klafter, Phys. Rep. 339 (2000) 1-77.

[17] E.W. Montroll, M.S. Shlesinger, in: Studies in Statistical Mechanics, Vol. 11, Eds. J. Lebowitz, E.W. Montroll (North-Holland, Amsterdam, 1984), pp. 1121.

[18] V.V. Uchaikin, V.M. Zolotarev, Chance and Stability (VSP, Utrecht, 1999).

[19] B.J. West, M. Bologna, P. Grigolini, Physics of Fractal Operators (Springer, New York, 2003)

[20] R. Gorenflo, F. Mainardi, Fractional Calculus: Integral and Differential Equations of Fractional Order, in Fractals and Fractional Calculus in Continuum Mechanics, Editors: A. Carpinteri and F. Mainardi. (Springer, Wien and New York 1997), pp. 223-276.

[21] R. Gorenflo, F. Mainardi, J. Comput. Appl. Math. 118 (2000) 283-299.

[22] F. Mainardi, Chaos, Solitons and Fractals, 7 (1996) 1461-1477.

[23] G.M. Zaslavsky, A.A. Stanislavsky, M. Edelman, Chaotic and Pseudochaotic Attractors of Perturbed Fractional Oscillator. Preprint: nlin.CD/0508018, to be published in Chaos.

[24] K.S. Miller, B. Ross, An Introduction to the Fractional Calculus and Fractional Differential Equations (Wiley, New York, 1993)

[25] I. Podlubny, Fractional Differential Equations (Academic Press, New York, 1999)

[26] H. Weitzner, G.M. Zaslavsky, Commun. Nonlin. Sci. and Numer. Simul. 8 (2003) 273-281; E-print: nlin.CD/0212024.

[27] V.E. Tarasov, G.M. Zaslavsky, Physica A 354 (2005) 249-261.

[28] A.V. Milovanov, J.J. Rasmussen, Phys. Lett. A 337 (2005) 75-80.

[29] S.G. Samko, A.A. Kilbas, O.I. Marichev, Fractional Integrals and Derivatives Theory and Applications (Gordon and Breach, New York, 1993)

[30] K.B. Oldham, J. Spanier, The Fractional Calculus (Academic Press, New York, 1974)

[31] I.M. Gelfand, G.E. Shilov, Generalized Functions I: Properties and Operations (Academic Press, New York, 1964)

[32] M. Caputo, Geophys. J. Roy. Astron. Soc. 13 (1967) 529-539.

[33] M. Caputo, F. Mainardi, Riv. Nuovo Cimento (Ser. II) 1 (1971) 161-198.

[34] A.A. Stanislavsky, Phys. Rev. E 70 (2004) 051103; Physica A 354 (2005) 101-110. 
[35] B.N.N. Achar, J.W. Hanneken, T. Clarke, Physica A 339 (2004) 311-319; Physica A 309 (2002) 275288.

[36] B.N.N. Achar, J.W. Hanneken, T. Enck, T. Clarke, Physica A 297 (2001) 361-367.

[37] A. Tofighi, Physica A 329 (2003) 29-34.

[38] Y.E. Ryabov, A. Puzenko, Phys. Rev. B 66 (2002) 184201.

[39] M.M. Dzherbashyan, Integral Transform Representations of Functions in the Complex Domain (Nauka, Moscow, 1966)

[40] A. Erdelyi, W. Magnus, F. Oberhettinger, F.G. Tricomi, Higher Transcendental Functions, Vol. 1. (Krieger, New York, 1981). Ch. 18. pp.206-227.

[41] H. Bateman, A. Erdelyi, Tables of Integral Transform Vol. 1. (McGraw-Hill, New York, 1954).

[42] M. Abramowitz, I.A. Stegun, (Eds.), "Sine and Cosine Integrals." Sec. 5.2 in Handbook of Mathematical Functions with Formulas, Graphs, and Mathematical Tables, 9th printing. (Dover, New York, 1972) pp. 231-233.

[43] R. Gorenflo, A.A. Kilbas, S.V. Rogosin, Integral Transform. Spec. Funct. 7 (1998) 215-224.

[44] R. Gorenflo, J. Loutchko, Y. Luchko, Fractional Calcul. and Appl. Anal. 5 (2002) 491-518.

[45] M.A. Leontovich, Izvestya Akademii Nauk SSSR, Ser. Fiz. 8 (1944) 16.

[46] J. Lighthill, Waves in Fluid (Cambridge Univ. Press, Cambridge, 1978) Sec. 3.7.

[47] B.B. Kadomtsev, Collective Phenomena in Plasmas (Pergamon, New York, 1978). Sec. 2.5.5. and 3.4.4.

[48] R.Z. Sagdeev, D.A. Usikov, G.M. Zaslavsky, Nonlinear Physics. From the Pendulum to Turbulence and Chaos (Harwood Academic, New York, 1988) Sec. 15.1.

[49] G.M. Zaslavsky, S.S. Abdullaev, Chaos 7 (1997) 182-186.

[50] W.A. Woyczynski, in Lévy Processes Theory and Applications, edited by O.E. BerndorffNielsen, et al. (Birkhäuser, Boston, 2001), pp.241-266.

[51] P. Biler, T. Funaki, W.A. Woyczynski, J. Diff. Eq. 148 (1998) 9-46. 